\title{
Recherches sur la résistance des sols aux maladies. X. - Comparaison de la mycoflore colonisant les racines de melons cultivés dans un sol résistant ou dans un sol sensible aux fusarioses vasculaires
}

Claude ALABOUVETTE, Yvonne COUTEAUDIER \& Jean LOUVET

I.N.R.A., Station de Recherches sur la Flore pathogène dans le Sol, 17, rue Sully, F 21034 Dijon Cedex

Ayant démontré précédemment que la population fusarienne du sol résistant de Châteaurenard est environ 10 fois plus importante que celle du sol sensible d'Ouroux, il convenait de comparer la colonisation fongique des racines de melons cultivés dans ces 2 sols. Une hypothèse avancée pour expliquer la résistance des sols de Châteaurenard stipulait en effet qu'une intense colonisation du rhizoplan par des Fusarium non pathogènes constituerait une barrière microbiologique interdisant l'accès du rhizoplan à l'agent pathogène. Les résultats acquis démontrent au contraire que la colonisation fongique de la racine est parfaitement comparable dans ces 2 sols, tant du point de vue quantitatif que qualitatif. La plante exerce sur la microflore tellurique un effet sélectif qui favorise la colonisation de la racine par les Fusarium spp. et, en particulier, par l'espèce $F$. oxysporum, indépendamment de l'importance des populations fusariennes des sols. La comparaison entre le niveau des populations de Fusarium spp. non pathogènes dans les sols et le niveau de la colonisation racinaire indique que les propagules de Fusarium ont une plus grande probabilité d'atteindre la surface de la racine dans le sol sensible que dans le sol résistant.

L'agent pathogène se comporte de la même façon que les Fusarium non pathogènes et les résultats montrent que, pour une densité d'inoculum identique, $F$. $o$. f. sp. melonis arrive plus souvent au contact de la racine dans le sol sensible que dans le sol résistant. Ainsi la compétition pour la conquête du rhizoplan apparaît-elle plus intense dans le sol résistant que dans le sol sensible. Mais c'est dans le sol lui-même, à proximité immédiate des racines, que se déroulent les phénomènes de compétition intragénérique qui limitent l'activité de l'agent pathogène dans le sol résistant.

Mots clés additionnels : Fusarium, Fusarium oxysporum $f . s p$. melonis, populations, compétition, rhizoplan.

Studies on disease suppressiveness of soils. X. - Comparison of the fungal microflora colonizing the roots of muskmelons growing in a wilt-suppressive and a wilt-conducive soil.

We previously demonstrated that the total population of Fusarium spp. was 10 times greater in the Châteaurenard soil (suppressive to Fusarium wilts) than in the Ouroux soil (conducive to Fusarium wilts). This fact prompted the hypothesis that wilt suppression was due to greater colonization of roots by non-pathogenic Fusarium spp. in the Châteaurenard than in the Ouroux soil, and that root colonization by non-pathogens acted as a microbial barrier to prevent contact between the pathogen and the root surface. The present study reveals, however, that root colonization by fungi was similar in suppressive and conducive soils, both quantitatively and qualitatively. The plant selectively favoured root colonization by Fusarium spp. and especially by Fusarium oxysporum, and colonization was unrelated to concentrations of Fusarium spp. in soil. Comparisons between the density of the non-pathogenic Fusarium spp. in the soils and their activity at the root surface showed that the probability for a Fusarium propagule to reach the root surface was greater in the conducive than in the suppressive soil. The pathogenic $F$. oxysporum $\mathrm{f}$. sp. melonis behaved in the same way as the non-pathogenic Fusarium; at the same inoculum density, propagules of the pathogen reached the root surface more often in the conducive than in the suppressive soil. These results indicate that competition may be more intense in the rhizosphere of plants growing in the suppressive than in the conducive soils.

Additional key words : Fusarium, Fusarium oxysporum $f . s p$. melonis, populations, competition, rhizoplane. 


\section{INTRODUCTION}

Dès les premières recherches consacrées à l'étude de la résistance des sols aux fusarioses vasculaires, nous avons remarqué l'importance de la flore fusarienne dans les sols de Châteaurenard. Les Fusarium non pathogènes représentent en effet 30 à 40 p. 100 de la mycoflore mise en évidence grâce aux techniques classiques de dénombrement sur milieu gélosé (ROUXEL et al., 1977). Nous avons ensuite démontré que ces Fusarium non pathogènes jouent un rôle indispensable dans l'expression de la résistance des sols aux fusarioses vasculaires. Il suffit en effet d'introduire des populations de $F$. oxysporum et de $F$. solani saprophytes dans le sol résistant préalablement traité à la chaleur pour y rétablir un bon niveau de résistance (ROUXEL et al., 1979 ; Louvet et al., 1981). Pour expliquer ce phénomène, nous avons émis l'hypothèse d'une compétition intragénérique qui, dans le sol résistant, limiterait l'installation ou le développement des $F$. oxysporum pathogènes. Dans l'article précédent (ALABOUvETTE et al., 1984), nous avons demontré que l'agent pathogène, introduit dans les sols résistant et sensible aux mêmes doses d'inoculum, s'y installe à des niveaux parfaitement comparables. En l'absence de plantes hôtes sensibles, la dynamique de la population pathogène est identique dans le sol résistant de Châteaurenard et dans le sol sensible d'Ouroux. Ces résultats montrent que les phénomènes de compétition intragénérique n'interdisent pas l'installation ni la conservation de l'agent pathogène dans le sol résistant. C'est pourquoi nous devons maintenant prendre en compte une autre hypothèse selon laquelle la compétition entre formes pathogènes et saprophytes de Fusarium se manifesterait pour la conquête de la racine de la plante-hôte (ROUXEL, 1978). Il est en effet permis de penser que l'abondance de la flore fusarienne du sol résistant conduit à une intense colonisation du rhizoplan par des Fusarium non pathogènes qui en interdiraient l'accès à l'agent pathogène, pourtant présent dans le sol.

Afin de vérifier cette hypothèse, nous avons analysé la mycoflore saprophyte colonisant les racines de melons cultivés dans les sols résistant et sensible en absence de l'agent pathogène. Puis, nous avons reproduit cette expérimentation en présence de $F$. $O$. f. sp. melonis afin de comparer le comportement de l'agent pathogène à celui des $F$. oxysporum non pathogènes.

\section{MATÉRIEL ET MÉTHODES}

\section{A. Analyse de la mycoflore des racines}

Des graines de melon, var. "Charentais", sont semées dans des pots contenant $500 \mathrm{ml}$ de sol résistant ou de sol sensible non infesté par l'agent pathogène. Après 3 semaines de culture, les plantules sont arrachées sous un jet d'eau de façon à ne pas endommager le système racinaire. A l'aide de ciseaux on élimine les racines secondaires pour ne conserver que la racine principale de chaque plante. Ces racines sont abondamment lavées à l'eau courante de manière à éliminer les particules de terre. Puis, nous inspirant de la technique des lavages successifs de HARLEY \& WAID (1955), nous avons réparti les racines en lots de 10 plantes que I'on soumet à des lavages à l'eau stérile suivis ou non d'une désinfection superficielle à l'hypochlorite de sodium (eau de javel à $7 \mathrm{p}$. 100 pendant $30 \mathrm{~s}$ ). Après ces traitements, les racines sont aseptiquement découpées en fragments de 2-3 mm de longueur qui sont déposés à la surface d'un milieu gélosé à base de malt. Cette expérimentation a été réalisée 2 fois.

\section{B. Recherche de l'agent pathogène à la surface des racines}

Afin de rechercher la présence de l'agent pathogène à la surface des racines de melons cultivés en sol résistant ou en sol sensible, nous avons reproduit la manipulation décrite ci-dessus, mais en utilisant des sols préalablement infestés avec une quantité déterminée (100 ou 2000 propagules $/ g$ de sol) de $F$. o. f. sp. melonis. Après 3 semaines de culture, les racines ont subi 3 lavages successifs à l'eau stérile avant d'être découpées en petits fragments déposés à la surface du milieu de culture. Afin de reconnaître l'agent pathogène, nous avons employé un mutant résistant au bénomyl dont le pouvoir pathogène et les activités saprophytiques en compétition ne diffèrent pas de la souche d'origine. Après $4 \mathrm{j}$ d'incubation, les colonies de $F$. oxysporum qui se sont développées sur le milieu nutritif à base de malt sont répertoriées et transplantées sur un milieu additionné de bénomyl. Après une semaine d'incubation, seules les colonies du mutant résistant au bénomyl se sont développées, ce qui permet d'estimer la proportion de l'agent pathogène parmi la population totale de $F$. oxysporum ayant colonisé la surface racinaire.

\section{RÉSULTATS}

Les 2 expériences réalisées successivement ont fourni des résultats semblables; nous les avons donc cumulés de façon à présenter de manière synthétique des pourcentages de fragments colonisés calculés à partir d'un grand nombre d'observations.

Le tableau 1 présente les pourcentages de fragments de racines qui ont donné naissance à une ou plusieurs colonies fongiques. Les résultats indiquent que la colonisation des racines par la flore fongique est légèrement plus importante en terre résistante qu'en terre sensible : les taux de colonisation sont respectivement 1,3 et 1,0 après lavage et 0,5 et 0,4 après désinfection. L'analyse des principaux groupes de champignons colonisant les racines ne fait pas apparaître de différence fondamentale sur le plan qualitatif entre le sol résistant et le sol sensible. Certains groupes dominent nettement par leur fréquence.

Après lavage, les Fusarium spp. sont présents sur 82 p. 100 des fragments de racines prélevées en terre résistante et sur 58 p. 100 en terre sensible. Ensuite, dans l'ordre de fréquence décroissant, viennent les Acremonium, les Mucor et les Pythium en terre résistante, les Penicillium, les Pythium et les Acremonium en terre sensible. Ce sont donc pratiquement les mêmes champignons qui colonisent préférentiellement la surface des racines du melon dans les 2 sols étudiés.

La désinfection superficielle élimine essentiellement les champignons qui ne sont pas capables de coloniser les tissus corticaux. La mycoflore est donc plus simple 


\section{TABLEAU 1}

Analyse de la mycoflore associée aux racines de melons cultivés en terre résistante ou sensible : pourcentages de fragments colonisés par les principales espèces fongiques après lavage ou désinfection superficielle de la racine.

Population of fungi colonizing the roots of muskmelons growing in suppressive and conducive soils: \% root pieces colonized by fungi after washing in sterile water or sturface-storilization with sodium hypochlorite.

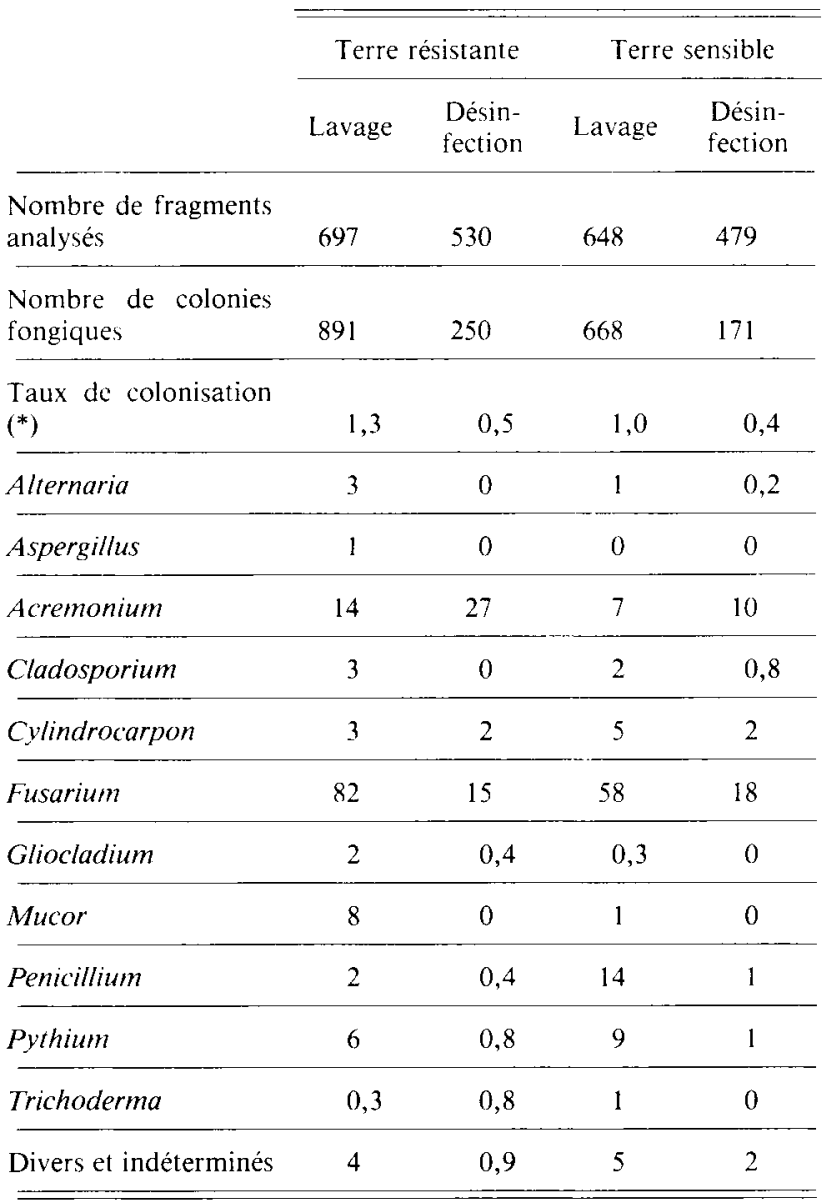

$\left(^{*}\right)$ rapport du nombre de colonies/nombre de fragments analysés.

et 2 groupes dominent nettement, ce sont les Fusarium spp. et les Acremonium spp. La proportion de fragments colonisés de façon profonde par les Fusarium est pratiquement la même en terre résistante $(15 \mathrm{p} .100)$ et en terre sensible (18 p. 100). Par contre, les Acremonium sont plus fréquents en terre résistante, où ils colonisent 27 p. 100 des fragments, qu'en terre sensible (10 p. 100). Ainsi, à l'exception des Acremonium, il n'apparaît pas de différence nette dans la colonisation interne des racines des plantes cultivées en terre résistante ou en terre sensible. Il semble donc que la colonisation fongique des racines du melon soit fonction de la plante elle-même plutôt que de la mycoflore du sol dans lequel elles se développent.

Pour mieux apprécier le rôle de la plante, nous avons comparé la proportion de chaque groupe de champignons dans la population fongique totale isolée du sol et dans la population fongique isolée des racines (tabl. 2). Les résultats font apparaître une nette différence entre terre sensible et terre résistante. Les Fusarium représentent 30 p. 100 de la mycoflore du sol résistant mise en évidence grâce à la méthode des suspensions-dilutions, 64 p. 100 de la flore racinaire de
TABLEAU 2

Pourcentages des principaux groupes de champignons isolés $d u$ sol et des racines $(\varepsilon=$ traces).

Comparison between the percentages of different fungi in suppressive and conducive soils, and on the roots of muskmelons after washing or surface-sterilization $(s=$ traces $)$.

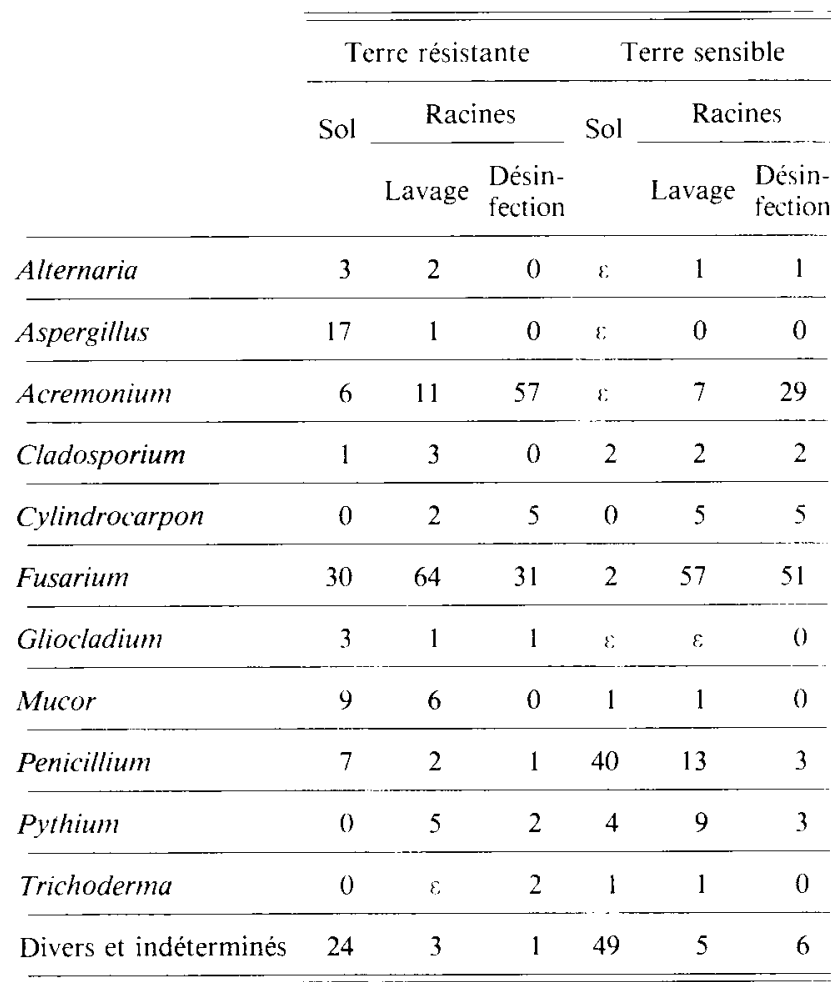

surface et 31 p. 100 de la mycoflore ayant pénétré dans les racines. En terre sensible, les Fusarium ne représentent que 2 p. 100 de la mycoflore totale du sol, mais 57 p. 100 de la flore racinaire superficielle et 51 p. 100 de la flore profonde des racines. L'influence de la plante apparaît très nettement en terre sensible où la proportion des Fusarium est 30 fois plus importante à la surface et dans les racines du melon que dans le sol lui-même. Ainsi, il semble que la colonisation des racines du melon soit réalisée préférentiellement par quelques espèces fongiques, indépendamment de l'importance initiale de ces espèces dans les sols.

Le rôle sélectif de la plante apparaît également au sein du genre Fusarium (tabl. 3). En effet, la fréquence relative des 3 principales espèces de Fusarium n'est pas la même dans les sols et au niveau des racines. Ce sont toujours les $F$. oxysporum qui dominent au niveau de la flore racinaire, en terre résistante comme en terre sensible, bien que dans la terre résistante elle-même, leur population soit très nettement inférieure à celle des autres espèces de Fusarium. Il apparaît donc clairement que des populations telluriques de $F$. oxysporum de niveau très différent $(1200 / \mathrm{g}$ en terre résistante et 180 en terre sensible) colonisent les racines de manière sensiblement identique. Par conséquent, la probabilité pour une propagule de $F$. oxysporum présente dans le sol d'atteindre la racine est beaucoup plus faible en terre résistante qu'en terre sensible. Cette observation conforte donc l'hypothèse d'une compétition intraspécifique pour la conquête des sites racinaires.

Ces résultats ayant été acquis dans des sols non infestés, il convient maintenant d'étudier le comportement 


\section{TABLEAU 3}

Comparaison entre le niveau des populations de Fusarium dans les sols résistant et sensible et les pourcentages de fragments de racines colonisés par les mêmes Fusarium.

Comparison between the population of Fusarium in suppressive and conducive soils and percentages of root pieces colonized by Fusarium spp.

\begin{tabular}{|c|c|c|c|c|c|}
\hline \multicolumn{3}{|c|}{ Terre résistante } & \multicolumn{3}{|c|}{ Terre sensible } \\
\hline \multirow{2}{*}{$\begin{array}{l}\text { Nbre de } \\
\text { propa- } \\
\text { gules } \\
\text { /g de } \\
\text { sol }\end{array}$} & \multicolumn{2}{|c|}{$\begin{array}{l}\% \text { de fragments de } \\
\text { racines colonisés }\end{array}$} & \multirow{2}{*}{$\begin{array}{c}\text { Nbre de } \\
\text { propa- } \\
\text { gules } \\
\text { /g de } \\
\text { sol }\end{array}$} & \multicolumn{2}{|c|}{$\begin{array}{l}\% \text { de fragments de } \\
\text { racines colonisés }\end{array}$} \\
\hline & Lavage & $\begin{array}{l}\text { Désin- } \\
\text { fection }\end{array}$ & & Lavage & $\begin{array}{l}\text { Désin- } \\
\text { fection }\end{array}$ \\
\hline
\end{tabular}

\begin{tabular}{lcccccc}
\hline $\begin{array}{l}\text { Fusarium } \\
\text { spp. }\end{array}$ & 10600 & 82 & 15 & 260 & 58 & 18 \\
\hline $\begin{array}{l}\text { F. oxyspo- } \\
\text { rum }\end{array}$ & 1200 & 37 & 8 & 180 & 39 & 11 \\
\hline $\begin{array}{l}\text { F. roseum } \\
\text { F. solani }\end{array}$ & 5600 & 36 & 5 & 40 & 10 & 3 \\
\hline \hline
\end{tabular}

de l'agent pathogène pour détérminer s'il est différent de celui des $F$. oxysporum non pathogènes.

La colonisation de la racine principale de melons, var. "Charentais », a été analysée après 3 semaines de culture dans les sols résistant et sensible infestés aux doses de 100 et 2000 propagules de $F$. $o$. f. sp. melonis par g de sol (tabl. 4). Dans cette expérience, le taux de colonisation des racines est, en terre sensible, un peu plus faible qu'en terre résistante. Cependant, les résul-

\section{TABLEAU 4}

Analyse de la mycoflore associée aux racines de melons cultivés en terre résistante et en terre sensible : pourcentages respectifs de Fusarium, $\mathbf{F}$. oxysporum et $\mathrm{F}$. o. $f$. $s p$. melonis isolés du rhizoplan, après lavage des racines à l'eau stérile.

Population of fungi colonizing the roots of muskmelons growing in suppressive and conducive soils : percentages of Fusarium spp., Fusarium oxysporum and $\mathrm{F}$. oxysporum $f$. sp. melonis isolated on the root-surface after 3 washings of the roots in sterile water.

\begin{tabular}{|c|c|c|c|c|}
\hline \multirow{2}{*}{$\begin{array}{l}\text { Dose d'infestation initiale en } \\
F . o . \text { melonis } \\
\text { Terres }\end{array}$} & \multicolumn{2}{|c|}{$\begin{array}{l}100 \text { germes } / g \\
\text { de sol }\end{array}$} & \multicolumn{2}{|c|}{$\begin{array}{c}2000 \text { germes } / g \\
\text { de sol }\end{array}$} \\
\hline & $\begin{array}{l}\text { Résis- } \\
\text { tante }\end{array}$ & $\begin{array}{l}\text { Sensi- } \\
\text { ble }\end{array}$ & $\begin{array}{l}\text { Résis- } \\
\text { tante }\end{array}$ & $\begin{array}{l}\text { Sensi- } \\
\text { ble }\end{array}$ \\
\hline $\begin{array}{l}\text { Nombre de fragments de raci- } \\
\text { nes analysés }\end{array}$ & 306 & 477 & 336 & 287 \\
\hline $\begin{array}{l}\text { Nombre de colonies fongi- } \\
\text { ques isolées }\end{array}$ & 318 & 336 & 464 & 275 \\
\hline Taux de colonisation & 1,0 & 0,70 & 1,4 & 1,0 \\
\hline$\frac{\text { Fusarium spp. }}{\text { Total colonies fongiques }} \times 100$ & 53 & 19 & 55 & 68 \\
\hline$\frac{\text { Fusarium oxysporum }}{\text { Fusarium spp. }} \times 100$ & 49 & 92 & 49 & 85 \\
\hline$\frac{\text { F. o. melonis }}{\text { F. oxysporum }} \times 100$ & 0 & 12 & 41 & 95 \\
\hline
\end{tabular}

tats restent comparables à ceux acquis précédemment (tabl. 1). Les Fusarium spp. constituent à eux seuls plus de 50 p. 100 des colonies fongiques isolées des fragments de racines. Dans le traitement où ils ne constituent que 19 p. 100 de la mycoflore, 2 autres genres, Acremonium et Cylindrocarpon, étaient très abondants et ont dû masquer ou inhiber le développement des Fusarium spp.

La prédominance de l'espèce $F$. oxysporum est confirmée ; elle représente près de 49 p. 100 des colonies fusariennes isolées des racines en terre résistante et 85 à 92 p. 100 dans le cas de la terre sensible.

La proportion de l'agent pathogène au sein de la population de $F$. oxysporum est très variable selon la dose initiale d'infestation et selon le sol colonisé.

Après un apport massif d'inoculum (2000 propagules/g de sol) juste avant le semis des melons, l'agent pathogène est mis en évidence à la surface des racines, aussi bien en sol résistant qu'en sol sensible. Cependant, il ne représente que 41 p. 100 de l'ensemble des colonies de $F$. oxysporum en terre résistante alors qu'il constitue la presque totalité ( 95 p. 100) des colonies isolées des racines des plantes cultivées en terre sensible. Dans ces conditions d'infestation particulièrement sévères, la maladie se manifeste en terre résistante, mais avec une intensité et une rapidité moins importantes qu'en terre sensible.

Lorsque l'agent pathogène est apporté aux sols à la dose de 100 propagules/g de sol, il n'est pas possible de le mettre en évidence à la surface des racines se développant dans la terre résistante. Aucune des colonies de $F$. oxysporum isolées n'appartient à la forme spéciale melonis. Au contraire, en terre sensible, l'agent pathogène a été détecté dans 12 p. 100 des cas, chaque isolement provenant d'une plante différente. Ces résultats sont en corrélation avec la gravité de la maladie qui, dans ces conditions d'infestation, ne se manifeste pas en terre résistante mais se développe en terre sensible. Ces résultats tendent à montrer que le comportement de l'agent pathogène n'est pas différent de celui des autres Fusarium. Introduites dans les sols à la même concentration, les propagules de $F$. $o$. f. sp. melonis ont une plus faible probabilité d'atteindre la surface racinaire en sol résistant qu'en sol sensible car elles sont confrontées à une population fusarienne totale beaucoup plus importante. D'autres expériences réalisées avec des densités d'inoculum intermédiaires entre 100 et 2000 germes $/ g$ de sol ont permis d'observer des situations intermédiaires dans lesquelles la proportion de $F . o$. f. sp. melonis au sein de la population totale de $F$. oxysporum est toujours beaucoup plus faible à la surface des racines se développant en terre résistante.

\section{DISCUSSION}

Les résultats acquis au cours de cette expérimentation indiquent que ce sont les mêmes espèces ou genres de champignons qui, avec des fréquences comparables, colonisent les racines de melon se développant en terre résistante ou en terre sensible. Cette observation est tout à fait comparable à celle de DAVET (1969) qui reconnaît une flore caractéristique des racines de tomate ou de haricot, indépendamment du sol dans lequel elles se développent. 
Les Fusarium constituent le groupe le plus important et parmi eux ce sont les $F$. oxysporum qui sont les plus abondants. L'abondance des $F$. oxysporum dans la mycoflore des racines de plantes variées a déjà été signalée par de nombreux auteurs (PETERSON, 1958; KUBIKOVA, 1963 ; TAYLOR \& PARKINSON, 1965 ; WARREN \& KOMMEDAHL, 1973a, $b, c)$. Cette espèce est considérée comme un colonisateur primaire du rhizoplan et du cortex racinaire. Dans le cas précis du melon, MEYER (1967) estime à 79 p. 100 la proportion de fragments de racines colonisés par les $F$. oxysporum. Nos résultats sont donc parfaitement concordants.

La fréquence des Acremonium, en particulier dans les racines formées en terre résistante, est à relier au fait que certains isolats de cette espèce possèdent la propriété de coloniser les vaisseaux de manière systémique. Nous l'avons vérifié, confirmant ainsi les observations de Kosswig (1955), Phillips et al. (1967), DAVET (1973).

La colonisation profonde des racines de melon ne présente donc aucun caractère particulier, que ce soit en terre résistante ou en terre sensible, et ce critère ne permet pas de différencier les 2 types de sol. L'hypothèse selon laquelle la colonisation des racines par les Fusarium saprophytes serait plus intense dans la terre résistante que dans la terre sensible n'est pas vérifiée.

Cependant, la comparaison du taux de colonisation des racines par les Fusarium avec le niveau de leurs populations dans les sols (tabl. 3) indique nettement que la probabilité de rencontre entre une propagule de Fusarium et la racine est beaucoup plus faible en terre résistante qu'en terre sensible. En effet, des populations de niveau très différent produisent une colonisation similaire de la surface racinaire. Il est clair que toutes les propagules n'ont pas chacune la possibilité de donner naissance à une microcolonie à la surface de la racine et cette probabilité est d'autant plus faible que le niveau de population est plus élevé.

L'agent pathogène ne manifeste pas un comportement différent de celui des Fusarium saprophytes. Introduit dans les sols résistant et sensible à des doses d'inoculum identiques, il s'établit dans ces 2 sols à des niveaux parfaitement comparables (ALABOUVETTE $e t$ al., 1984). Cependant, les résultats acquis au cours de cette étude montrent que, pour une même densité d'inoculum, l'agent pathogène atteint plus fréquemment la surface de la racine dans le sol sensible que dans le sol résistant (tabl. 4). F.o.f. sp. melonis, confronté dans le sol résistant à une population fusarienne beaucoup plus importante que dans le sol sensible, y subit donc une compétition plus intense et arrive moins fréquemment au contact de la racine.
Ainsi, ces résultats semblent indiquer que les phénomènes de compétition intragénérique se déroulent dans le sol lui-même plutôt qu'à la surface des racines et qu'ils diminuent la probabilité de conquête de la surface racinaire par l'agent pathogène.

Différents arguments viennent conforter cette interprétation de nos résultats. Nous avons déjà montré que $F$. o. f. sp. melonis ne perd pas son pouvoir pathogène dans le sol résistant (LOUVET et al., 1976 ; ALAßBOUVETTE et al., 1979). Il conserve donc ses propriétés spécifiques qui lui confèrent un avantage décisif sur les $F$. oxysporum saprophytes dès qu'il colonise la racine. A ce niveau, seuls des phénomènes de prémunition pourraient limiter l'expression de la maladie. Mais cette hypothèse, déjà envisagée et rejetée par ROUXEL (1978), ne semble pas devoir être retenue dans la mesure où la colonisation interne des racines par des $F$. oxysporum non pathogènes est tout aussi importante en terre sensible qu'en terre résistante. D'autre part, nous avons déjà indiqué à plusieurs reprises (LOUVET et al., 1976 ; ALABOUVETTE et al., 1982) que la résistance des sols de Châteaurenard n'est pas absolue, mais que la maladie s'y manifeste lorsque les doses d'inoculum introduites sont très élevées. Ainsi, lorsque la probabilité d'un contact direct entre l'agent pathogène et la surface racinaire est importante, aucun autre mécanisme se situant à la surface ou à l'intérieur des racines n'est capable d'empêcher la contamination ou l'infection des plantes. Les mécanismes responsables de la résistance du sol de Châteaurenard se déroulent donc dans le sol lui-même avant la phase de pénétration de l'agent pathogène dans le cortex racinaire.

L'ensemble de cette expérimentation et les résultats acquis antérieurement (ALABOUVETTE et al., 1984) conduisent à proposer l'hypothèse suivante : des phénomènes de compétition intragénérique, se déroulant dans le sol à proximité immédiate de la racine, limiteraient le développement de l'agent pathogène dans le sol résistant. Ces mécanismes apparaissent d'autant plus efficaces que la population fusarienne totale du sol est plus importante et que la proportion de l'agent pathogène au sein de cette population est plus faible. Ces phénomènes de compétition limiteraient la germination des chlamydospores et le développement des tubes germinatifs en direction de la racine. Certaines observations préliminaires déjà publiées (ALABOUVETTE et al., 1979 ; ALABOUVETTE et al., 1980) confortent cette hypothèse qui doit cependant faire l'objet de nouvelles investigations.

\begin{abstract}
Alabouvette C., 1983. La réceptivité des sols aux Fusarioses vasculaires, rôle de la compétition nutritive entre microorganismes. Thèse Doct. ès-Sci. Nat., Univ. Nancy, 158 p.

Alabouvette C., Rouxel F., Louvet J., 1979. Characteristics of Fusarium-wilt suppressive soils and prospects for their utilization in biological control. In Schippers B. \& Gams W. : Soil-Borne Plant Pathogens, 165-182. Acad. Press.
\end{abstract}

Alabouvette C., Rouxel F., Louvet J., 1980. Recherches sur la résis- tance des sols aux maladies. VII. Etude comparative de la germination des chlamydospores de Fusarium oxysporum et Fusarium solani au contact des sols résistant et sensible aux fusarioses vasculaires. Ann. Phytopathol., 12 (1), 21-30.

Alabouvette C., Couteaudier Y., Louvet J., 1982. Comparaison de la réceptivité de différents sols et substrats de culture aux fusarioses vasculaires. Agronomie, 2 (1), 1-6.

Alabouvette C., Couteaudier Y., Louvet J., 1984. Recherches sur la 
résistance des sols aux maladies. IX. - Dynamique des populations de Fusarium spp. et de Fusarium oxysporum f. sp. melonis dans un sol résistant et un sol sensible aux fusarioses vasculaires. Agronomie, $4(8)$.

Davet P., 1969. Observations sur la mycoflore des racines de quelques plantes maraîchères du Liban. Rev. Mycol., 34, 62-78.

Davet P., 1973. Distribution et évolution du complexe parasitaire des racines de tomate dans une région du Liban ou predomine Pvreno-

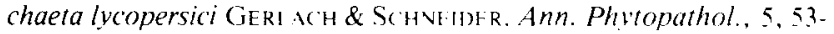
63.

Harley J. L., Waid J. S., 1955. A method of studying active mycelia on living roots and other surfaces in the soil. Trans. Br. Mycol. Soc., 38, 104-118.

Kosswig W., 1955. Die Svmptomatologie der Fusarium-welken der Gurke (Cucumis sativus L.) und ihr Verhältnis zu den Welkekrankheiter anderer Pflanzen. Paul PAREY, Hamburg und Berlin, 148 p.

Kubikova J., 1963. The surface mycoflora of ash roots. Trans. Br. Mycol. Soc., 46, 107-114.

Louvet J., Rouxel F., Alabouvette C.., 1976. Recherches sur la resistance des sols aux maladies. I. Mise en evidence de la nature microbiologique de la résistance d'un sol au développement de la fusariose vasculaire du melon. Ann. Phytopathol., 8, 425-436.

Louvet J., Alabouvette C., Rouxel F., 1981. Microbiological suppressiveness of some soils to Fusarium-wilts. In Nisison P. E., Totssoun T. A., Cook R. J. Fusarium : Diseases, Biology and Taxonomy. Pa State Univ. Press, Univ. Park and London, $457 \mathrm{p}$.

Meyer J. A., 1967. Recherches sur les Fusarioses. H. Ecologic et pathogénie du Fusarium oxysporum. Ann. Epiphyties, 18 (2), 241-247.
Peterson E. A., 1958. Observations of fungi associated with plant roots. Can. J. Microbiol., 4, 257-265.

Phillips D. V., Leben C., Allison C. C., 1967. A mechanism for the reduction of Fusarium wilt by a Cephalosporium species. Phytopathology, 57, $916-919$.

Rouxel F., 1978. Etude de la résistance microbiologique des sols au. Fusarioses vasculaires. Application aux sols de la Basse Vallée de la Durance. Thèse Doct. ès-Sci., Univ. Dijon, 134 p.

Rouxel F., Alabouvette C., Louvet J., 1977. Recherches sur la résistance des sols aux maladies. II. Incidence de traitements thermiques sur la résistance microbiologique d'un sol à la fusariose vasculaire du melon. Ann. Phytopathol., 9, 183-192.

Rouxel F., Alabouvette C., Louvet J., 1979. Recherches sur la résislance des sols aux maladies. IV. Mise en évidence du rôle des Fusaritum autochtones dans la résistance d'un sol à la fusariose vasculaire du melon. Ann. Phytopathol., 11, 199-207.

Taylor (. S. . Parkinson D.. 1965. Studies on fungi in the root region 11. rungl associated with the roots of Phaseolas vialgarts L. Plant \& Soil., 22, 1-20.

Warren H. L., Kommedahl T., $1973 a$. Fertilization and wheat refuse effects on Fusarium species associated with wheat roots in Minnesota. Phylopathology, 63, 103-108.

Warren H. I.., Kommedahl T., 1973b. Prevalence and pathogenicity to corn of tusaram species from corn rools, rhzosphere, ressdues and soil. Phytopathology, 63, 1288-1290.

Warren H. L., Kommedahl T., 1973c. Root-infecting species of Fusurium in soil and in the roots, rhizosphere and residues of oats. Phytopathology, 63, 1401-1403. 